\title{
ORIGINAL ARTICLE Extended follow-up and impact of high-risk prognostic factors from the phase 3 RESONATE study in patients with previously treated CLL/SLL
}

\author{
JR Brown ${ }^{1,23}$, P Hillmen ${ }^{2,23}$, S O'Brien ${ }^{3}$, JC Barrientos ${ }^{4}$, NM Reddy ${ }^{5}$, SE Coutre ${ }^{6}$, CS Tam $^{7}$, SP Mulligan $^{8}$, U Jaeger $^{9}$, PM Barr $^{10}$,

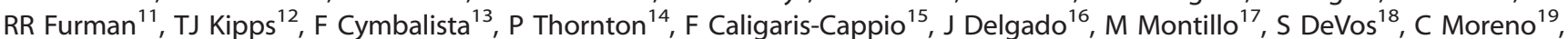

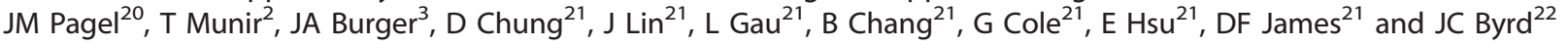

In the phase 3 RESONATE study, ibrutinib demonstrated superior progression-free survival (PFS), overall survival (OS) and overall response rate (ORR) compared with ofatumumab in relapsed/refractory CLL patients with high-risk prognostic factors. We report updated results from RESONATE in these traditionally chemotherapy resistant high-risk genomic subgroups at a median follow-up of 19 months. Mutations were detected by Foundation One Heme Panel. Baseline mutations in the ibrutinib arm included TP53 (51\%), SF3B1 (31\%), NOTCH1 (28\%), ATM (19\%) and BIRC3 (14\%). Median PFS was not reached, with 74\% of patients randomized to ibrutinib alive and progression-free at 24 months. The improved efficacy of ibrutinib vs ofatumumab continues in all prognostic subgroups including del17p and del11q. No significant difference within the ibrutinib arm was observed for PFS across most genomic subtypes, although a subset carrying both TP53 mutation and del17p had reduced PFS compared with patients with neither abnormality. Reduced PFS or OS was not evident in patients with only del17p. PFS was significantly better for ibrutinibtreated patients in second-line vs later lines of therapy. The robust clinical activity of ibrutinib continues to show ongoing efficacy and acceptable safety consistent with prior reports, independent of various known high-risk mutations.

Leukemia (2018) 32, 83-91; doi:10.1038/leu.2017.175

\section{INTRODUCTION}

The last decade has seen remarkable advances in therapy of chronic lymphocytic leukemia (CLL) with the advent of combination chemotherapy (fludarabine and cyclophosphamide $(F C))^{1,2}$ and then chemoimmunotherapy with rituximab (FCR). ${ }^{3,4}$ Indeed, a subset of low-risk patients with IGHV-mutated disease may be essentially cured by FCR, but still may suffer the toxic effects of chemotherapy. However, patients with higher-risk genetic abnormalities (IGHV unmutated) experience inferior outcomes with a median progression-free survival (PFS) of 4.2 years, ${ }^{5}$ while those with the highest-risk factor, del(17)(p13.1) [del17p], have a median PFS of $\sim 1$ year. ${ }^{3}$ Shorter remissions particularly in high-risk subgroups, and poor survival with conventional salvage regimens after FCR relapse, ${ }^{6,7}$ present a need for novel therapies for CLL.

Bruton's tyrosine kinase (BTK) has emerged as an attractive target with the observation that the genetic disease, X-linked agammaglobulinemia, in which BTK is inactivated, results in the failure of B cells to develop, with few other complications outside of infectious morbidity that is clinically manageable. ${ }^{8}$ Ibrutinib is a once-daily, first-in-class, covalent inhibitor of BTK, which binds irreversibly to Cys481 in BTK with an $\mathrm{IC}_{50}$ of $0.5 \mathrm{~nm}^{9}$ allowing for once-daily, oral administration with sustained enzyme inhibition. RESONATE was a randomized comparison of ibrutinib to ofatumumab in previously treated CLL patients, many with highrisk prognostic factors. The first report of this trial demonstrated that ibrutinib significantly improved PFS, overall survival (OS), and overall response rates (ORR) compared with ofatumumab, and was acceptably tolerated, ${ }^{10}$ leading to ibrutinib's approval for previously treated CLL and del17p CLL.

Ibrutinib shows marked efficacy in genetically high-risk CLL, particularly del17p, ${ }^{11}$ known to confer a very poor prognosis in addition to TP53 mutation. ${ }^{12}$ Whole exome sequencing studies have identified recurrent mutations particularly in $\mathrm{NOTCH} 1,{ }^{13}$ ATM, SF $3 B 1^{14,15}$ and BIRC3. ${ }^{16}$ These mutations, enriched in previously treated CLL, are associated with poor prognosis in retrospective studies. ${ }^{17}$ In the CLL8 study, both TP53 and SF3B1 mutations were risk factors for reduced PFS, but only TP53 for reduced OS, ${ }^{18}$ and NOTCH1 mutation was associated with lack of benefit from the addition of CD20 monoclonal antibody in two studies. ${ }^{18,19}$ In retrospective studies, NOTCH1 mutation has also been associated with increased risk of Richter transformation (RT). ${ }^{20,21}$ Here, we report updated results with up to 2-year follow-up from the

\footnotetext{
${ }^{1}$ Dana-Farber Cancer Institute, Boston, MA, USA; ${ }^{2}$ St James Institute of Oncology, Leeds, UK; ${ }^{3}$ MD Anderson Cancer Center, Houston, TX, USA; ${ }^{4}$ Hofstra Northwell School of Medicine, Hempstead, NY, USA; ${ }^{5}$ Vanderbilt-Ingram Cancer Center, Nashville, TN, USA; ${ }^{6}$ Stanford University School of Medicine, Stanford, CA, USA; ${ }^{7}$ Peter MacCallum Cancer Centre and St Vincent's Hospital, Melbourne, Australia; ${ }^{8}$ Royal North Shore Hospital, Sydney, Australia; ${ }^{9}$ Medical University of Vienna, Vienna, Austria; ${ }^{10}$ University of Rochester Cancer Center, Rochester, NY, USA; ${ }^{11}$ Weill Cornell Medical College/New York Presbyterian Hospital, New York, NY, USA; ${ }^{12}$ UCSD Moores Cancer Center, San Diego, CA, USA; ${ }^{13}$ Hôpital Avicenne, Paris, France; ${ }^{14}$ Beaumont Hospital, Dublin, Ireland; ${ }^{15}$ Universita Vita-Salute San Raffaele, Milan, Italy; ${ }^{16} \mathrm{Hospital}$ Clinic, Barcelona, Spain; ${ }^{17}$ Niguarda Cancer Center Niguarda Hospital, Milan, Italy; ${ }^{18}$ David Geffen School of Medicine at UCLA, Los Angeles, CA, USA; ${ }^{19}$ Hospital de la Santa Creu Sant Pau, Barcelona, Spain; ${ }^{20}$ Swedish Cancer

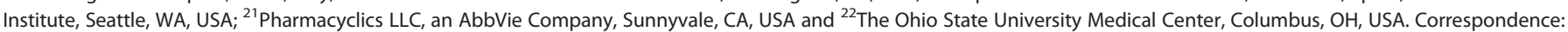
Dr JR Brown, Division of Hematologic Malignancies, Dana-Farber Cancer Institute, 450 Brookline Ave, Boston, MA 02215, USA.

E-mail: Jennifer_Brown@dfci.harvard.edu
}

${ }^{23}$ Co-lead authors.

Received 17 February 2017; revised 11 May 2017; accepted 23 May 2017; accepted article preview online 8 June 2017; advance online publication, 21 July 2017 
RESONATE trial, including subgroup analysis for baseline high-risk genetic features. We find that the marked benefit of ibrutinib continues and is preserved in all evaluable genetic subgroups.

\section{MATERIALS AND METHODS}

Study design

RESONATE is a multicenter, international, open-label, randomized, phase 3 study that compares the efficacy and safety of ibrutinib to ofatumumab, as previously described (NCT01578707). ${ }^{10}$ At the time of this update, 123 patients randomized to ofatumumab received ibrutinib in crossover. See Supplementary Appendix for details.

\section{Patients}

All patients provided written informed consent. The study was approved by the institutional review board or ethics committee at each participating institution and conducted in accordance with the Declaration of Helsinki and the International Conference on Harmonization Guidelines for Good Clinical Practice.

Procedures

Updated efficacy data from this analysis, including PFS and ORR, were by investigator assessment. Procedural details have been described previously ${ }^{10}$ and can be found in Supplementary Appendix.

\section{Mutation Identification}

In order to make our results applicable to general practice, all mutation analyses were performed by a clinically available, FDA-approved targeted next generation sequencing panel that is being increasingly used in clinical practice, the Foundation One Heme Panel. This panel has a median sequencing depth $500 \mathrm{X}$ and reports variant allele frequencies of approximately $1 \%$ or higher ${ }^{22}$ (see Supplementary Appendix for details). As is typical in clinical practice, matched germline testing was not performed; known common polymorphisms were removed based on those identified in the 1000 Genomes Project (dbSNP135). Putative mutations were evaluated based on COSMIC v62; all candidate mutations of any detected allele frequency, classified as known, likely, or unknown, were counted as a mutation. Of note, 138 of 140 TP53 mutations identified were classified as known or likely to have a functional effect.

Statistical analysis

Please see Supplementary Appendix for additional details on outcomes measures. ${ }^{10}$

\section{RESULTS}

Baseline patient characteristics

Patients in the ibrutinib $(n=195)$ and ofatumumab $(n=196)$ arms had a median of three and two prior therapies, respectively, with 53 vs $46 \%$ of patients receiving study therapy in the fourth line of therapy or beyond; $18 \%$ of ibrutinib- and $27 \%$ of ofatumumabtreated patients had received only one prior therapy (Table 1); approximately $32 \%$ of ibrutinib patients had del $17 p$, and $32 \%$ had del(11)(q22.3) [del11q].

Consistent with this relapsed higher-risk population, the frequencies of TP53, NOTCH1, SF3B1 and BIRC3 mutations were high compared with other studies. ${ }^{16,18,23}$ Most notably, $51 \%$ of ibrutinib and $46 \%$ of ofatumumab patients carried mutations in TP53. Of 195 ibrutinib patients, 154 had TP53 samples available. Of 45 ibrutinib patients with del17p and a sample available for analysis, 84\% $(n=38)$ had coexistent TP53 mutation, consistent with prior reports, ${ }^{24}$ of 79 ibrutinib patients with mutated TP53, $52 \%(n=41)$ carried this mutation in the absence of del17p. In the ibrutinib arm, 39 of 153 (25\%) evaluable patients had complex karyotype (CK); of 47 del17p patients in the ibrutinib arm with karyotype data reported, 20 (43\%) had CK.
Table 1. Baseline characteristics

\begin{tabular}{|c|c|c|}
\hline Characteristic & $\begin{array}{l}\text { Ibrutinib } \\
(\mathrm{n}=195)\end{array}$ & $\begin{array}{l}\text { Ofatumumab } \\
\quad(\mathrm{n}=196)\end{array}$ \\
\hline Median age, years (range) & $67(30-86)$ & $67(37-88)$ \\
\hline$\geqslant 70$ years, $n(\%)$ & $40 \%$ & $41 \%$ \\
\hline Male, $n(\%)$ & $66 \%$ & $70 \%$ \\
\hline Rai stage III/IV, n (\%) & $56 \%$ & $58 \%$ \\
\hline $\begin{array}{l}\text { Median number of prior therapies } \\
\text { (range), } n(\%)\end{array}$ & $3(1-12)$ & $2(1-13)$ \\
\hline 1 & $18 \%$ & $27 \%$ \\
\hline 2 & $29 \%$ & $27 \%$ \\
\hline$\geqslant 3$ & $53 \%$ & $46 \%$ \\
\hline Del17p, $n / N(\%)$ & $63 / 195(32 \%)$ & $64 / 196(33 \%)$ \\
\hline Del11q, $n / N(\%)$ & $63 / 190(33 \%)$ & $59 / 191(31 \%)$ \\
\hline Trisomy $12, n / N(\%)$ & $22 / 138(16 \%)$ & $27 / 145(19 \%)$ \\
\hline Complex karyotype, $n / N$ (\%) & $39 / 153(25 \%)$ & $33 / 147(22 \%)$ \\
\hline $\mathrm{CD} 38(\geqslant 30 \%), n / N(\%)$ & $69 / 160(43 \%)$ & $69 / 155(45 \%)$ \\
\hline \multicolumn{3}{|l|}{ IGHV, n/N (\%) } \\
\hline Unmutated & $98 / 134(73 \%)$ & $83 / 132(63 \%)$ \\
\hline Mutated & $36 / 134(27 \%)$ & $49 / 132(37 \%)$ \\
\hline Gene mutations, n (\%) & $\begin{array}{l}\text { Ibrutinib } \\
(\mathrm{n}=154)^{\mathrm{a}}\end{array}$ & $\begin{array}{l}\text { Ofatumumab } \\
\qquad(\mathrm{n}=149)^{\mathrm{a}}\end{array}$ \\
\hline \multicolumn{3}{|l|}{ ATM } \\
\hline Mutated & $30(19 \%)$ & $33(22 \%)$ \\
\hline Not mutated & $124(81 \%)$ & $116(78 \%)$ \\
\hline \multicolumn{3}{|l|}{ NOTCH1 } \\
\hline Mutated & $43(28 \%)$ & $45(30 \%)$ \\
\hline Not mutated & $111(72 \%)$ & $104(70 \%)$ \\
\hline \multicolumn{3}{|l|}{$S F 3 B 1$} \\
\hline Mutated & 47 (31\%) & $44(30 \%)$ \\
\hline Not mutated & 107 (69\%) & $105(70 \%)$ \\
\hline \multicolumn{3}{|l|}{ TP53 } \\
\hline Mutated & 79 (51\%) & $68(46 \%)$ \\
\hline Not mutated & 75 (49\%) & $81(54 \%)$ \\
\hline \multicolumn{3}{|l|}{ MYD88 } \\
\hline Mutated & $3(2 \%)$ & $3(2 \%)$ \\
\hline Not mutated & $151(98 \%)$ & $146(98 \%)$ \\
\hline \multicolumn{3}{|l|}{ BIRC3 } \\
\hline Mutated & $21(14 \%)$ & $15(10 \%)$ \\
\hline Not mutated & $133(86 \%)$ & $134(90 \%)$ \\
\hline
\end{tabular}

Patient disposition

With a median 19-month follow-up (maximum 26 months on study), 145 (74\%) patients on the ibrutinib arm continue ibrutinib on study. Fifty (26\%) patients discontinued ibrutinib (19 (10\%) due to progressive disease (PD), $13(7 \%)$ due to adverse events (AE) and $10(5 \%)$ due to death; Supplementary Table 1).

\section{Efficacy}

Ibrutinib showed significant improvement in PFS compared with ofatumumab (median not reached (NR) vs 8.1 months; HR 0.106; Figure 1a) with the benefit of ibrutinib apparent for all clinical and genetic subgroups (Supplementary Figure 1). Median PFS was NR on the ibrutinib arm in any prognostic subgroup including del17p (Figure 1b) and del11q. Second-line ibrutinib PFS outcomes were significantly improved compared with those in later lines of therapy $(P=0.0348)$ (Figure $1 \mathrm{c}$ ). Supplementary Figure 2 shows PFS for ibrutinib by one, two, three and $\geqslant$ four prior therapies. 
a

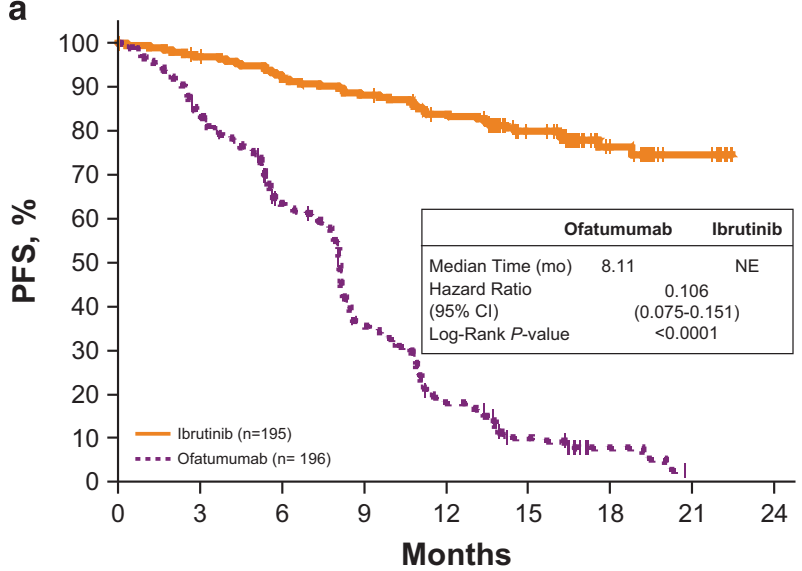

C

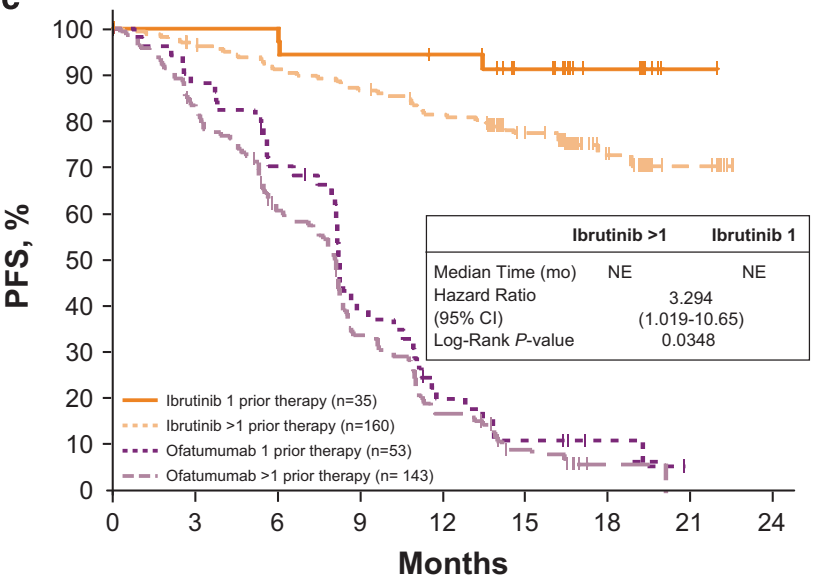

b

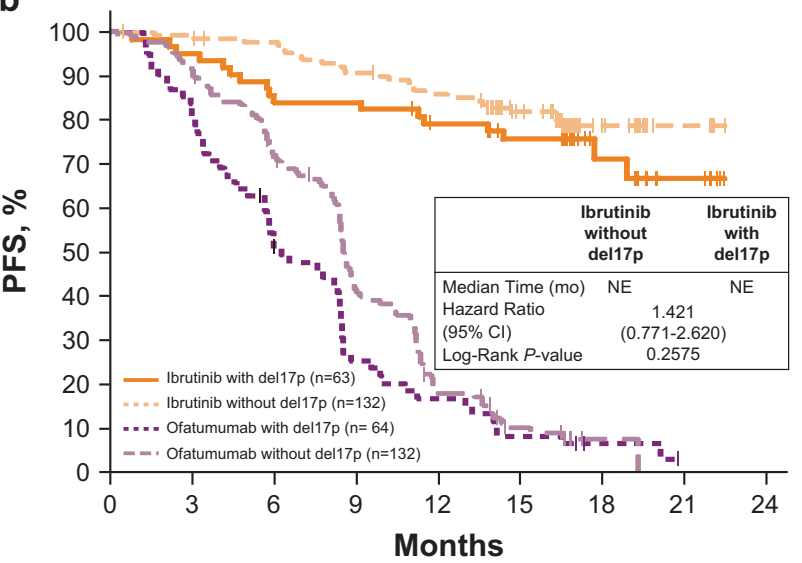

d

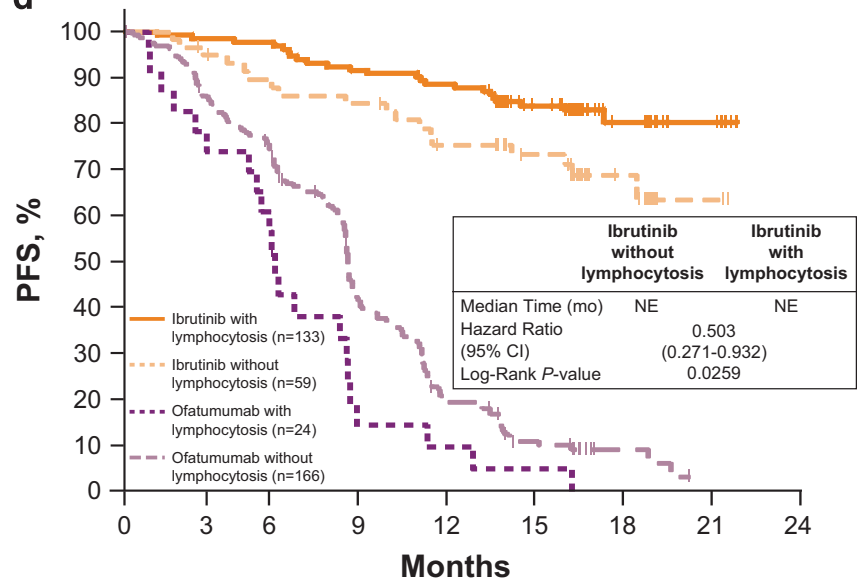

Figure 1. PFS in patient subgroups. (a) Overall PFS; $P<0.0001$ for the comparison of ibrutinib vs ofatumumab. (b) PFS in patients with del17p $\mathrm{CLL} ; P=0.2575$ for ibrutinib-treated patients with and without del17p and $P=0.0582$ for ofatumumab-treated patients with and without del17p. (c) PFS in patients with 1 vs $>1$ prior therapy; $P=0.0348$ for ibrutinib-treated patients with 1 prior line vs $>1$ prior line of therapy and $P=0.2761$ for ofatumumab-treated patients with 1 prior line vs $>1$ prior line of therapy. (d) PFS in patients who did or did not develop lymphocytosis; $P=0.0259$ for ibrutinib-treated patients with and without lymphocytosis at baseline and $P=0.0095$ for ofatumumab-treated patients with and without lymphocytosis.

The 24 -month PFS rate was $74 \%$ for the ibrutinib arm. The 18-month PFS with ibrutinib was similar regardless of baseline genetic factors including unmutated IGHV, del17p, del11q, CK or mutations including NOTCH1, BIRC3 and ATM (Table 2). With SF3B1 mutation, a trend toward a lower 18-month PFS rate was seen in the ibrutinib arm $(P=0.1424)$; however, when all data were taken into account by log-rank test $(P=0.3349)$, this trend was less apparent. The presence of del17p or del11q did not confer inferior PFS outcomes compared with PFS in patients with neither deletion $(P=0.2160$; Figure 2a). Those with any del17p/TP53 alteration (carrying either one or both abnormalities) did not show significantly inferior PFS compared with those with neither abnormality $(P=0.1306$; Figure 3a). Interestingly, however, an exploratory subset analysis focusing on patients with both del17p and TP53 mutation $(n=38)$ compared with patients with neither of these abnormalities $(n=68)$ showed worse PFS in the subset carrying both abnormalities $(P=0.0381$; Figure 2b). Although survival assessment by CK status was somewhat limited due to missing CK data in $22 \%$ of patients, available data suggest that PFS was not significantly different in ibrutinib patients with known CK relative to those without CK (Figure $3 \mathrm{~b}$ ). Among ofatumumabtreated patients, unmutated IGHV $(P=0.0436)$ and presence of del11q $(P=0.0654)$ had a lower 18 -month PFS rate (Table 2$)$ and NOTCH1 mutation was associated with a worse overall PFS (logrank $P=0.0064)$; conversely, the presence of a NOTCH1 mutation did not negatively impact the efficacy of ibrutinib (Table 2; Supplementary Table 2).

A limited multivariate Cox proportional hazards regression analysis (MVA) was performed including the following candidate factors: age, Rai stage, ECOG, number of prior therapies, del11q, del17p, $\beta 2$-microglobulin, and disease refractory to purine analogues. Gene mutations (for example, TP53 alone) and CK were not included in the MVA due to missing data in $\sim 23 \%$ of patients in the ibrutinib and ofatumumab arms. Del17p, del11q and 32 -microglobulin were considered significant prognostic factors for PFS in all patients. No significant prognostic factors were identified with current follow-up when the MVA was limited to the ibrutinib arm.

Eighty-six percent of patients randomized to ibrutinib were alive at the time of analysis. Of patients randomized to ofatumumab, $77 \%$ were alive with the majority of these patients $(108 / 196)$ continuing crossover therapy with ibrutinib. Crossover was instituted $\sim 4$ months after the last patient was randomized, and OS analyses showed consistent benefit with ibrutinib in the naïve intent-to-treat comparison and when OS was adjusted for crossover (based on rank preserving structural failure time model methodology), with a significant reduction in the risk of death of approximately $64 \%$ for ibrutinib vs ofatumumab (Supplementary Figure 3). OS was similar between ibrutinib subgroups including del17p without del11q, del11q without del17p, neither deletion (Supplementary Figure 4) and del17p and/or TP53 (Figure 4a for 
Table 2. 18-month PFS rate and ORR by subgroup

\begin{tabular}{|c|c|c|c|c|}
\hline \multirow[t]{2}{*}{ Subgroup } & \multicolumn{2}{|c|}{ 18-month $P F S^{\mathrm{a}}$} & \multicolumn{2}{|c|}{$O R R, \mathrm{n} / \mathrm{N}(\%)^{\mathrm{a}, \mathrm{d}}$} \\
\hline & Ibrutinib ( $\mathrm{N}=195)$ & Ofatumumab $(\mathrm{N}=196)$ & Ibrutinib $(\mathrm{N}=195)$ & Ofatumumab $(\mathrm{N}=196)$ \\
\hline \multicolumn{5}{|c|}{ Median number of prior therapies } \\
\hline 1 & $91 \%^{\mathrm{c}}$ & $11 \%$ & $35 / 35(100 \%)^{c}$ & $14 / 53(26 \%)$ \\
\hline 2 & $76 \%^{c}$ & $0 \%$ & $141 / 160(88 \%)^{c, b}$ & $35 / 143(24 \%)^{b}$ \\
\hline \multicolumn{5}{|l|}{ Del11q } \\
\hline Yes & $83 \%$ & $0 \%$ & $57 / 63(90 \%)$ & $7 / 59(12 \%)^{c}$ \\
\hline No & $73 \%$ & $10 \%$ & $114 / 127(90 \%)$ & $42 / 132(32 \%)^{c}$ \\
\hline \multicolumn{5}{|l|}{ Del17p } \\
\hline Yes & $71 \%$ & $7 \%$ & $56 / 63(89 \%)$ & $13 / 64(20 \%)^{\mathrm{g}}$ \\
\hline No & $79 \%$ & $8 \%$ & $120 / 132(91 \%)$ & $36 / 132(27 \%)^{g}$ \\
\hline Trisomy 12 & $77 \%$ & $0 \%$ & $21 / 22$ (95\%) & $8 / 27(30 \%)$ \\
\hline$C D 38(\geqslant 30 \%)$ & $78 \%$ & $0 \%$ & $65 / 69$ (94\%) & $21 / 69$ (30\%) \\
\hline \multicolumn{5}{|l|}{ IGHV } \\
\hline Unmutated & $77 \%$ & $0 \%$ & $90 / 98(92 \%)$ & $22 / 83(27 \%)$ \\
\hline Mutated & $74 \%$ & $15 \%$ & $32 / 36(89 \%)$ & $12 / 49(24 \%)$ \\
\hline Gene mutations & Ibrutinib ( $\mathrm{N}=154)$ & Ofatumumab $(\mathrm{N}=149)$ & Ibrutinib ( $\mathrm{N}=154)$ & Ofatumumab $(\mathrm{N}=149)$ \\
\hline \multicolumn{5}{|l|}{ ATM } \\
\hline \multicolumn{5}{|l|}{ TP53 } \\
\hline Mutated & $66 \%^{f}$ & 0 & $72 / 79$ (91\%) & $13 / 68(19 \%)^{\mathrm{g}}$ \\
\hline Not mutated & $81 \%^{f}$ & $9 \%$ & $69 / 75(92 \%)$ & $27 / 81(33 \%)^{g}$ \\
\hline \multicolumn{5}{|l|}{ MYD88 } \\
\hline Mutated & 0 & 0 & $2 / 3(67 \%)$ & $1 / 3(33 \%)$ \\
\hline Not mutated & $73 \%$ & $8 \%$ & $139 / 151$ (92\%) & $39 / 146(27 \%)$ \\
\hline \multicolumn{5}{|l|}{ BIRC3 } \\
\hline Mutated & $81 \%$ & 0 & $20 / 21(95 \%)$ & $4 / 15(27 \%)$ \\
\hline Not mutated & $72 \%$ & $9 \%$ & $121 / 133(91 \%)$ & $36 / 134(27 \%)$ \\
\hline
\end{tabular}

any alteration vs none, log-rank $P=0.1903)$. No significant difference in OS benefit was observed for ibrutinib-treated patients with respect to known $\mathrm{CK}$, although data were limited $(P=0.1610$; Figure $4 \mathrm{~b})$. The OS 18 -month point estimate was $86 \%$ in the ibrutinib arm: $83 \%$ for the del17p subgroup, $89 \%$ for the del11q subgroup, $85 \%$ for those with neither deletion, and $79 \%$ for those with CK. There was a trend towards improved OS for patients on the ibrutinib arm treated in a second-line setting (HR $2.874, P=0.1324)$ compared with those salvaged in later lines of therapy (Supplementary Figure 5).

Lymphocytosis occurred in $69 \%$ of ibrutinib-treated patients and was typically transient, resolving with continued ibrutinib 


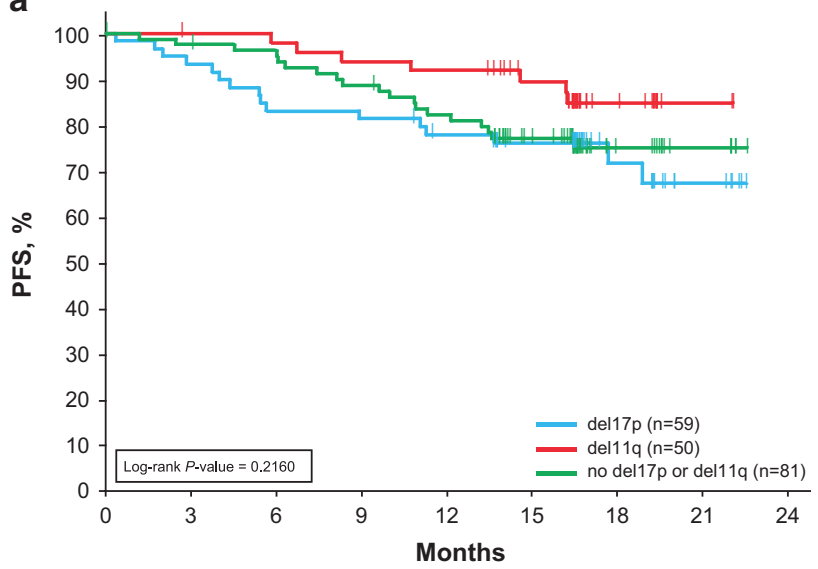

b

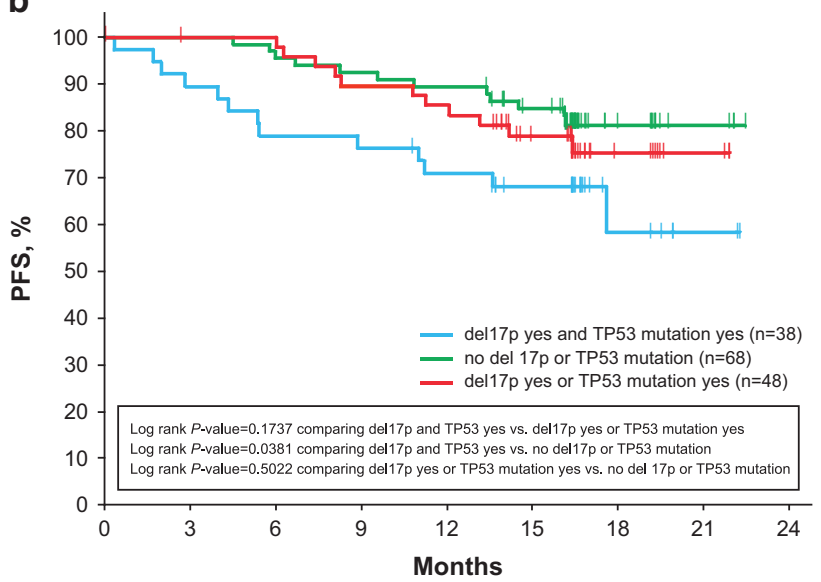

Figure 2. PFS with ibrutinib by cytogenetics (FISH)/mutational association. (a) PFS in patients by del17p/del11q CLL subgroups; the del17p subgroup contains patients with del17p with or without del11q ( $P=0.2160$ comparing all three groups). (b) PFS in patients by del17p/TP53 CLL subgroups ( $P=0.1737$ for both del17p and TP53 mutation vs either del17p or TP53 mutation; $P=0.0381$ for both del17p and TP53 mutation vs neither; $P=0.5022$ for either del17p or TP53 mutation vs no del17p or TP53 mutation).

therapy in $87 \%$ of patients with a median duration of 14.1 weeks. PFS appeared better for those who developed lymphocytosis vs those who did not (Figure 1d); a previous study has reported noninferior outcomes between patients with or without persistent lymphocytosis. ${ }^{25}$ Ibrutinib patients with mutated IGHV showed a slight trend to greater initial increase in absolute lymphocyte count (ALC) compared with those with unmutated IGHV (Supplementary Figure 6), with apparent slower resolution in patients with mutated IGHV vs those with unmutated IGHV similar to a previous report $(P<0.001){ }^{26}$

The best ORR increased in the ibrutinib arm with $90 \%$ of patients now attaining PR with lymphocytosis (PR-L) or better, as compared with 83 vs $23 \%$ (ibrutinib vs ofatumumab, $P<0.0001$ ) by investigator assessment at interim analysis. ${ }^{10}$ ORR was higher in the ibrutinib arm in all evaluable subgroups compared with ofatumumab $(P<0.0001)$. In addition, the complete response (CR) rate improved with $7 \%$ of patients demonstrating CR (9 (5\%)) or complete response with incomplete blood count recovery (CRi; 4 (2\%)) compared with $2 \%$ at interim analysis. The latest CR/CRi was achieved in the ibrutinib arm at 17.5 months. One patient on ofatumumab achieved CR at 8 months. The ORR was $100 \%$ in ibrutinib-treated patients with only one prior therapy as compared with $88 \%$ with $\geqslant 2$ prior therapies $(P=0.0275$, Table 2$)$. No a

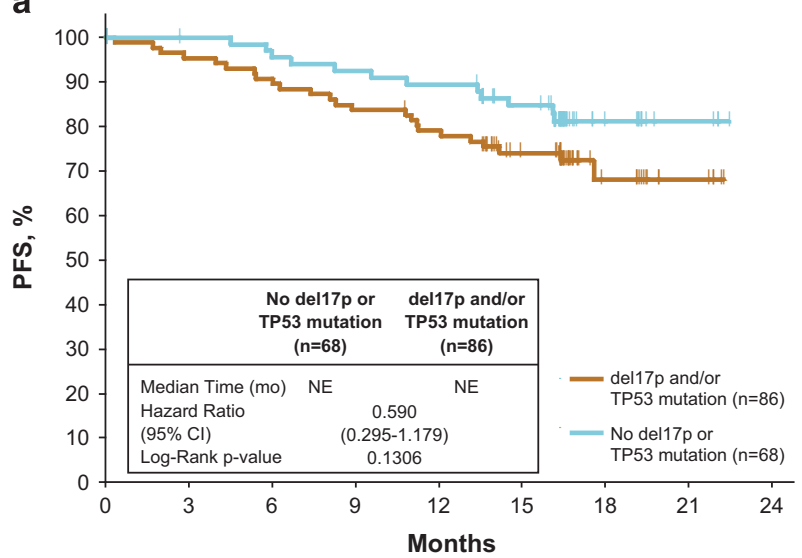

b

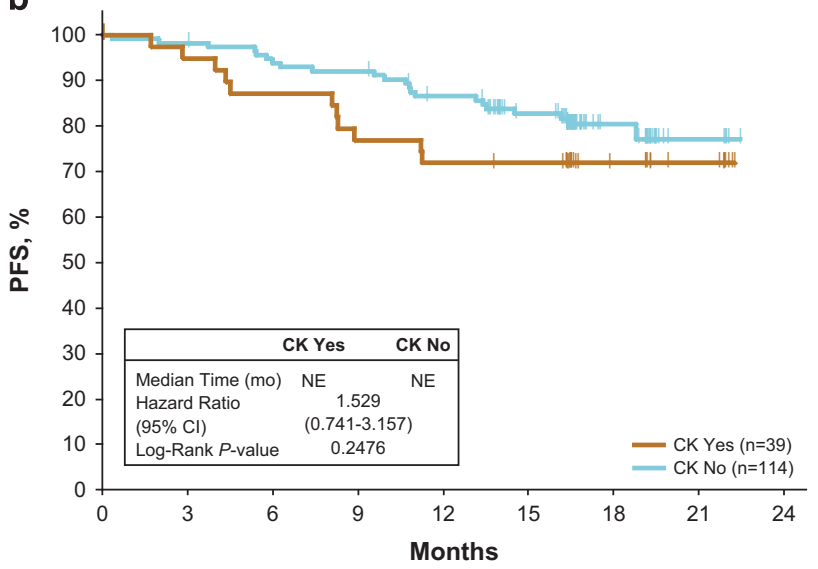

Figure 3. PFS with ibrutinib by del17p/TP53 status and complex karyotype. (a) PFS with ibrutinib in patients by any del17p/TP53 alteration vs none. (b) PFS with ibrutinib in all patients with or without complex karyotype.

difference was seen in ORR within the ibrutinib arm among patients with or without various genetic mutations. On the ofatumumab arm, patients with del11q and CK had a significantly lower ORR compared with those without del11q and CK ( $P=0.0038$ and $P=0.0015$, respectively) (Table 2 ).

Twenty-eight ibrutinib patients progressed including patients who discontinued due to $\mathrm{AE}$ and later progressed. Of the 28 patients who progressed, 20 had CLL progression (without RT) and 8 had RT, of which 6 developed large cell lymphoma and 2 Hodgkin disease (5 ofatumumab patients progressed with RT, including 1 after crossover to ibrutinib). Clonal relationship of the RT to prior CLL was unknown. Of the 8 ibrutinib-treated patients with RT (6 within 1 year, 2 within two years), baseline mutations were available for 6 patients and reported as follows: NOTCH1 $(n=2), \operatorname{BIRC} 3(n=1), \operatorname{TP53}(n=5), \operatorname{SF3B1}(n=1)$, or ATM $(n=1)$. Other high-risk features were observed in these RT patients, including del17p $(n=4)$, del11q $(n=2)$, and CK $(n=3)$, and in nonRT PD patients $(n=20)$ including del17p $(n=9)$, del11q $(n=4)$, CK $(n=5)$, NOTCH1 $(n=7)$, TP53 $(n=11)$, SF3B1 $(n=10)$. At the time of analysis, 12 of 28 patients who progressed had died (including 8 patients with non-RT progression and 4 with RT). Seventeen of 28 patients had received subsequent anticancer therapy (including 11 patients with non-RT progression and 6 with RT). The most common subsequent therapies were regimens containing ofatumumab $(n=6), \mathrm{R}-\mathrm{CHOP}(n=4)$ or $\mathrm{R} \pm \mathrm{EPOCH}(n=4)$. Of 14 patients who discontinued ibrutinib due to $\mathrm{PD}$ and received subsequent anticancer therapy, 6 had RT: 2 died, and 3 were alive at time of analysis on subsequent therapy (See Supplementary Table 3 for 
a

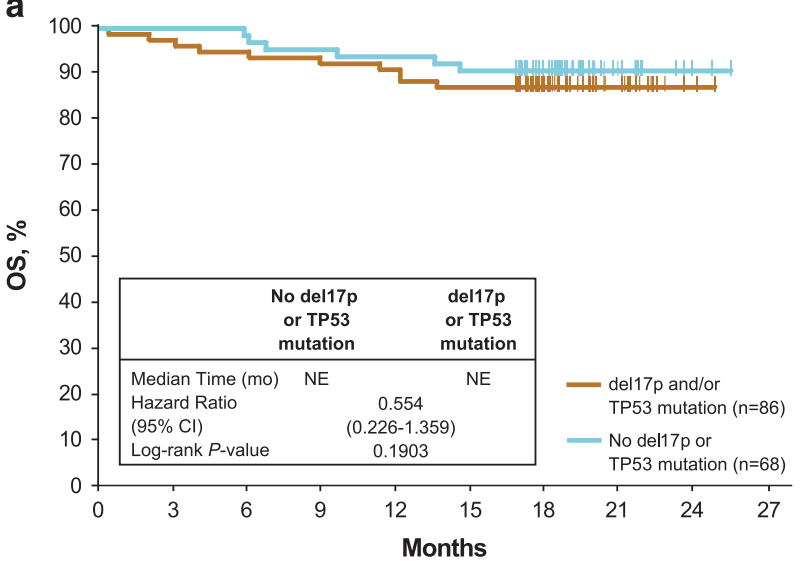

b

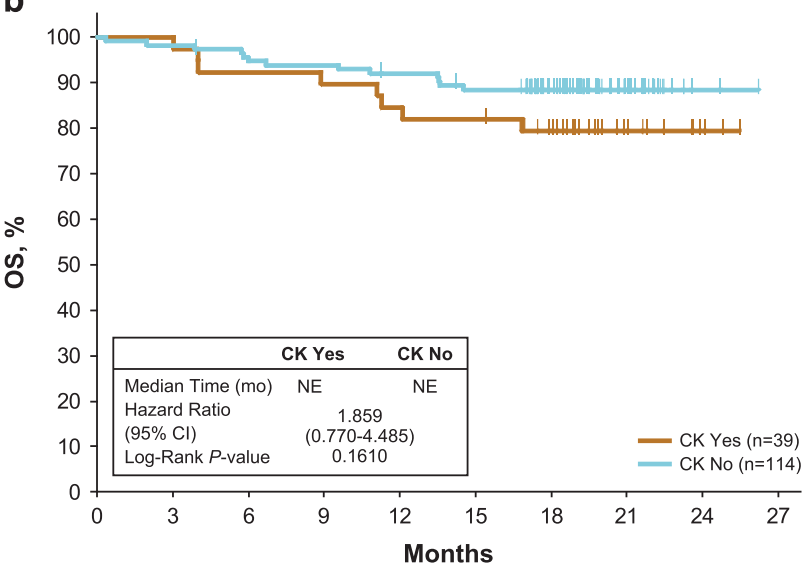

Figure 4. Overall survival with ibrutinib by del17p/TP53 status and complex karyotype. (a) Overall survival with ibrutinib in patients by any del17p/TP53 alteration vs none. (b) Overall survival with ibrutinib in all patients with or without complex karyotype. details). Eight with non-RT PD received subsequent anticancer therapy, and 3 were alive at analysis.

\section{Safety}

The longer-term safety profile of ibrutinib in this randomized phase 3 study was consistent with the 3-year follow-up from the phase 2 study. ${ }^{27}$ The most common cumulative AE of any grade remains diarrhea, now 54 from $48 \%$ at interim analysis, followed by fatigue ( 34 vs $28 \%$ ), nausea (31 vs $26 \%$ ) and fever (30 vs $24 \%$ ) (Table 3). The most common (>10\%) infections included upper respiratory tract infections $(25 \%$ any grade, $0.5 \%$ grade $\geqslant 3$ ), sinusitis $(19 \%$ any grade, $0.5 \%$ grade $\geqslant 3)$, pneumonia $(17 \%$ any grade, $12 \%$ grade $\geqslant 3)$, and urinary tract infections $(14 \%$ any grade, $4 \%$ grade $\geqslant 3$ ). Pneumocystis jirovecii pneumonia (grade 3 ) occurred in 1 patient (0.5\%). Seven patients $(4 \%)$ had grade 5 infections, which included pneumonia $(n=4)$, sepsis $(n=2)$ and neutropenic sepsis $(n=1)$ (Supplementary Table 1); none of these grade 5 infections were considered related to study treatment. Nineteen patients (10\%) in the ibrutinib arm had received prophylactic growth factor support, and 131 patients (67\%) had received some form of anti-infective prophylaxis, most commonly with antiviral agents including aciclovir $(n=53 ; 27 \%)$ and valaciclovir $(n=52 ; 27 \%)$. The most common antibiotic agents used for prophylaxis included trimethoprim-sulfamethoxazole $(n=47 ; 24 \%)$ and levofloxacin $(n=16 ; 8 \%)$.

New onset of diarrhea, atrial fibrillation (AF), arthralgia and grade $\geqslant 3$ infection decreased over time (Table 4 ). Grade $\geqslant 3$ bleeding events were infrequent with three additional events over approximately 1-year follow-up since interim analysis, including grade 3 epistaxis and spontaneous psoas hematoma (the latter during concomitant enoxaparin and clopidogrel) and grade 4 subdural hematoma (post-traumatic event, resolved and patient restarted and continues ibrutinib). Any grade AF occurred in 13 (7\%) patients (grade $1(n=2)$, grade $2(n=4)$, grade $3(n=7))$ with the median time to onset of first event being 5.1 months). Eight $(62 \%)$ of these patients resolved their AF event (median 3 days to resolution, range $1-42$ ), and all received thromboprophylaxis that

\begin{tabular}{|c|c|c|c|c|c|c|}
\hline Diarrhea & 93 (47.7) & $105(53.8)$ & $8(4.1)$ & $9(4.6)$ & 0 & 0 \\
\hline Pyrexia & 46 (23.6) & $58(29.7)$ & $3(1.5)$ & $3(1.5)$ & 0 & 0 \\
\hline Cough & 38 (19.5) & $51(26.2)$ & 0 & $1(0.5)$ & 0 & 0 \\
\hline Neutropenia & $42(21.5)$ & $50(25.6)$ & $32(16.4)$ & $38(19.5)$ & 0 & 0 \\
\hline Anemia & 44 (22.6) & $49(25.1)$ & $9(4.6)$ & $12(6.2)$ & 0 & 0 \\
\hline $\begin{array}{l}\text { Upper respiratory } \\
\text { tract infection }\end{array}$ & 31 (15.9) & $49(25.1)$ & $1(0.5)$ & $1(0.5)$ & 0 & 0 \\
\hline Peripheral edema & $22(11.3)$ & $38(19.5)$ & 0 & 0 & 0 & 0 \\
\hline Headache & $27(13.8)$ & $33(16.9)$ & $2(1.0)$ & $3(1.5)$ & 0 & 0 \\
\hline Pneumonia & $19(9.7)$ & $33(16.9)$ & $13(6.7)$ & $20(10.3)$ & $3(1.5)$ & $4(2.1)$ \\
\hline Thrombocytopenia & $33(16.9)$ & $33(16.9)$ & $11(5.6)$ & $11(5.6)$ & 0 & 0 \\
\hline Vomiting & $28(14.4)$ & $33(16.9)$ & 0 & 0 & 0 & 0 \\
\hline
\end{tabular}

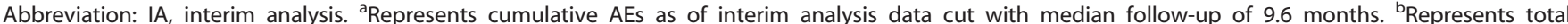
cumulative AEs (including all IA data) with median follow-up of 19 months, maximum follow-up of 24 months. 
Table 4. Adverse events of interest for ibrutinib-treated patients by time of event onset

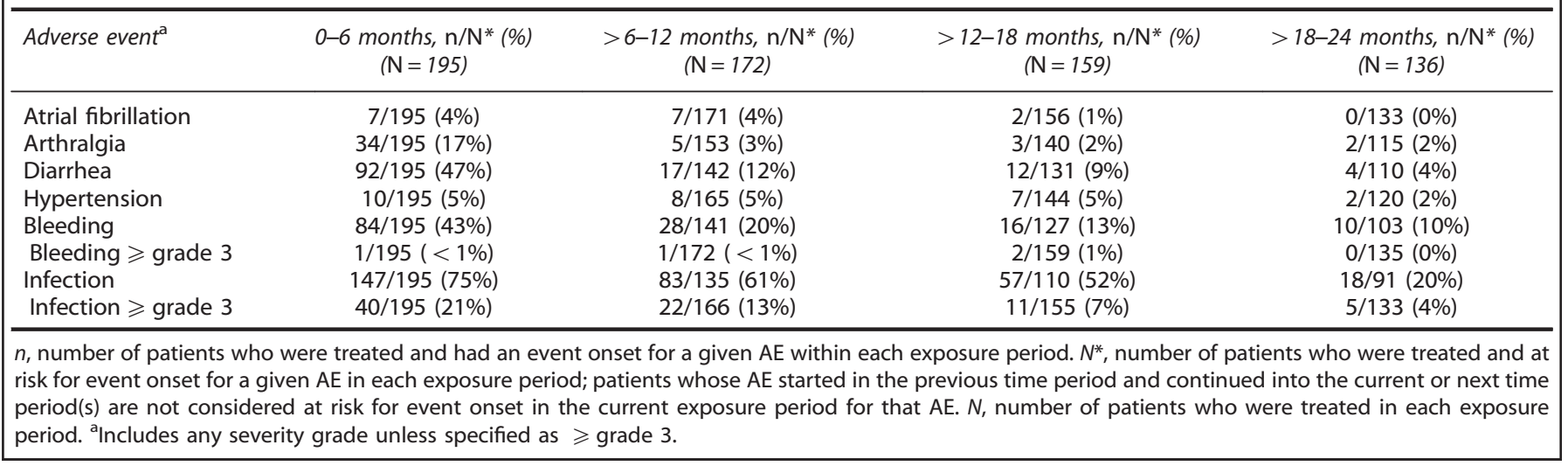

included aspirin, low molecular weight heparin, direct oral anticoagulants or combinations thereof (Supplementary Table 4). One patient with AF had a major hemorrhagic event that was the grade 3 spontaneous psoas hematoma described above.

\section{DISCUSSION}

Efficacy with ibrutinib remained high at this 2-year follow-up of the RESONATE study, with $74 \%$ of patients alive and progressionfree. Patients on the ibrutinib arm treated after only one prior therapy experienced improved efficacy compared with those salvaged after $\geqslant$ second-line therapy. Phase 3 data in treatmentnaive patients without del17p also suggest high efficacy in the frontline setting with significantly improved PFS and OS compared with chlorambucil with a median 19-month follow-up. ${ }^{28}$ Taken together, the results from this study as well as from studies of ibrutinib in frontline therapy ${ }^{27,28}$ suggest that the best results occur when ibrutinib has been used earlier in the treatment course. This observation has also been true of other therapies historically, including chemoimmunotherapy with FCR, perhaps reflecting the accumulation of multiple and broad mechanisms of drug resistance over time with treatment. The outcome of ongoing randomized trials using standard combination chemoimmunotherapy vs ibrutinib-containing regimens in the upfront setting will be required to determine the true extent of ibrutinib benefit in that setting as compared with the early relapsed setting.

Tolerability to ibrutinib was well maintained in this relapsed patient population with extended treatment. The most common side effects were similar to those at interim analysis with only a slight increase in the cumulative event rate despite the additional year of therapy. Specifically, the rate of new infection, diarrhea and arthralgia appeared lower later in follow-up when compared with the first 6 months, consistent with a recent report of long-term follow-up with ibrutinib at the Ohio State University, in which patients who discontinued for reasons other than progression did so relatively early, followed by a plateau. ${ }^{29}$ Severe (grade $\geqslant 3$ ) bleeding events were infrequent with three events occurring during the additional follow-up period, with two of these events occurring in the context of concomitant anticoagulation and in a post-traumatic setting, respectively. Recent studies have reported reduced ristocetin- ${ }^{30}$ and collagen- ${ }^{31}$ mediated platelet aggregation in patients treated with ibrutinib. Grade $\geqslant 3$ bleeding events with ibrutinib were uncommon in these reports. With regard to AF, the median time of onset of 5.1 months in our study was similar to 3.8 months reported in the largest cohort of ibrutinib-treated CLL patients with AF $(n=56) .^{32}$ Further, hypertension has been noted throughout the ibrutinib treatment course including at later times. $^{27,28}$ Thus, ongoing monitoring of toxicity, particularly in patients who may remain on ibrutinib for many years, remains important.

Prior non-randomized trials have reported that RT tends to occur early in the course of ibrutinib treatment ${ }^{29,33,34}$ followed by a plateau in incidence. In this study, which followed patients only until disease progression, more events of RT were reported in the ibrutinib arm compared with the ofatumumab arm (8 patients vs 5 patients, respectively, with 6 and 4 patients with RT in the first year of follow-up), but these frequencies reflect a median PFS (and therefore of follow-up) of 8.1 months on ofatumumab vs ongoing follow-up in most patients on ibrutinib. Previous reports of three randomized controlled CLL trials have identified a similar incidence of RT on the ibrutinib and control arms; there were 3 patients with RT on the placebo arm vs 0 on the ibrutinib arm of HELIOS ${ }^{35} 1$ RT on the chlorambucil arm vs 0 on the ibrutinib arm of RESONATE- $2{ }^{28}$ and 2 RT cases on each arm of RESONATE at time of primary analysis. ${ }^{10}$ Moreover, the $4 \%$ rate of RT in the ibrutinib arm of our current analysis is not different from historical rates reported with other CLL regimens. ${ }^{36-38}$ Patients who developed RT in the ibrutinib arm had frequent adverse genomic features at baseline, including del17p, NOTCH1, and TP53 abnormalities, which are known to be associated with, and may contribute to, the development of RT. Thus, the risk of RT with ibrutinib treatment does not appear to be higher compared with other treatment regimens in CLL, and likely reflects the natural history of high-risk, relapsed/refractory disease.

For the first time, we report randomized efficacy outcomes with ibrutinib in high-risk FISH-defined genetic subgroups in addition to more novel high-risk gene mutations. Ibrutinib markedly improved PFS and ORR in all genetic subgroups over ofatumumab. In particular, with an additional year of follow-up, patients on ibrutinib with either del17p or TP53 mutation did not show markedly worse PFS than those without these genetic abnormalities, although in an exploratory analysis, the presence of both abnormalities was associated with a decrease in the PFS curve. Notably, similar to prior reports, ${ }^{39}>1 / 2$ of patients with TP53 mutations did not have del17p - such patients experience poor outcomes to chemotherapy regimens, ${ }^{40}$ yet TP53 mutational testing is not standardly performed in the United States. The 3 -year update of the phase 2 data demonstrated that patients with del17p have significantly lower PFS (median 28 months) than patients with del11q or neither of these anomalies, where the median PFS was NR. ${ }^{27}$ In that study, TP53 mutation was not tested, but it is likely that most patients with del17p had TP53 mutation, as previously reported; ${ }^{24}$ thus, these findings are likely consistent with the emerging decrease in PFS observed in the current study for patients with both abnormalities. In a subsequent efficacy analysis of patients with del17p from the same study, patients without CK appeared to experience the most favorable PFS/OS 
outcomes. ${ }^{41}$ Consistent with these findings, investigators at MD Anderson Cancer Center and Ohio State University have previously reported that CK adversely affects ibrutinib outcomes. ${ }^{29,42}$ This finding was not observed yet in this study, but may be due to incomplete data or still relatively short follow-up.

Analysis of other relevant prognostic genes including SF3B1, $B I R C 3, A T M$ and NOTCH1 revealed no difference in progression outcomes between ibrutinib patients with or without these mutations at 19 months follow-up. In contrast, NOTCH1-mutated patients randomized to ofatumumab fared significantly worse than their non-mutated counterparts, perhaps consistent with previous reports in which NOTCH1 mutations were associated with lack of benefit of CD20 antibody therapies. ${ }^{18,19}$ It should be noted that, in order to make our results relevant to clinical practice, we used a clinically available next generation sequencing panel applied only to tumor, and because of that, the analysis may be limited by the absence of comparison to matched germline, which may result in some rare germline variants being counted as somatic mutations. In addition, all mutations detected in the NGS panel, which has a detection limit of approximately $1 \%$ allele frequency, were included in the clinical analysis, yet at present, it is unknown whether a cutoff should be applied for a minimum allele frequency when assessing these mutations in relation to clinical outcome. Given this uncertainty, we chose to err on the side of including any detected candidate mutation, particularly as prior literature does suggest that even tiny TP53 mutated clones are associated with poor outcome. ${ }^{39,43}$ Ultimately, detailed scientific studies with large uniformly treated datasets will be required to determine whether an allele frequency cutoff would be more appropriate.

In this study, patients who developed lymphocytosis experienced longer PFS compared with those who did not. In a 3-year follow-up of treatment-naïve and relapsed/refractory patients with CLL/SLL, Byrd et al. reported similar PFS outcomes in patients with and without persistent lymphocytosis at one year of ibrutinib therapy. ${ }^{27}$ Woyach et al. have also reported similar outcomes among patients with or without persistent lymphocytosis treated with ibrutinib, although a trend towards improved PFS was seen in patients who achieved PR-L. ${ }^{25}$ An association between prolonged lymphocytosis and favorable prognostic features was also observed, with ibrutinib responders with prolonged treatmentrelated lymphocytosis more likely to carry favorable prognostic markers including del13q and mutated IGHV. ${ }^{25}$ Taken together, these data suggest that prolonged lymphocytosis is associated with favorable prognostic factors that are associated with longer response, but ultimately longer follow-up is warranted to establish a definite correlation.

Ultimately, given the low number of PFS events in patients treated with ibrutinib, the follow-up is still too short to definitively know whether subgroups characterized by higher-risk mutations, including del17p, will have reduced PFS. Meanwhile, the majority of relapsed CLL patients treated on this trial continue to do extremely well, underscoring the significant impact of ibrutinib in altering the course of relapsed CLL.

\section{CONFLICT OF INTEREST}

JRB has received honoraria from Celgene, Gilead, Infinity, Genentech/Roche, Janssen, Pharmacyclics, Pfizer and Sub BioPharma, has served in an advisory role for Celgene, Gilead, Infinity, Genentech, Janssen, Pfizer and Pharmacyclics, and has been reimbursed for expenses by Gilead, Sun BioPharma, Pfizer and Janssen. PH has served as a consultant for Roche, Glaxo Smith Kline, Janssen, Gilead, and AbbVie, received honoraria from AbbVie, Gilead, Glaxo Smith Kline, Roche, Novartis, Pharmacyclics and Janssen, and research funding from Roche, Novartis, Glaxo Smith Kline, Janssen, Gilead, AbbVie, Celgene, Pharmacyclics. SOB has served as a consultant and received honoraria from Pharmacyclics and Janssen, and received research funding from Pharmacyclics. JCBarrientos has served as a consultant to Gilead, AbbVie, and Janssen, and received research funding from AbbVie and Gilead.
NMR has served as a consultant for Celgene, Infinity, Gilead, and AbbVie. SEC has served in an advisory role for Janssen and Pharmacyclics, and has received research funding from AbbVie and Pharmacyclics. CT has received honoraria from Janssen, research funding from Janssen, and has served in an advisory role for Janssen. SPM has served as a consultant for and received honoraria from AbbVie, Gilead, Glaxo Smith Kline, Janssen, Roche, received research funding from AbbVie, Janssen, Roche, participated in speakers' bureau for AbbVie, Gilead, Janssen, Roche. UJ has served as a consultant, and received honoraria and reimbursements from Janssen and Roche. PMB has served as a consultant for Pharmacyclics, AbbVie and received research funding from Pharmacyclics. RRF has received honoraria from Pharmacyclics, and has served in an advisory role and on Speakers' Bureaus for Pharmacyclics. TJK has served as a consultant for AbbVie, Genentech, Gilead, and received research funding from AbbVie, Genentech, Pharmacyclics. FC has received research funding from Janssen, honoraria from Gilead, Janssen, Mundipharma and AbbVie, has consulted for Gilead, Janssen and AbbVie, and been reimbursed by Janssen, and Roche. PT has served as a consultant for Janssen. FCC has received honoraria and served as a consultant for Celgene, Janssen, Pharmacyclics. JD has received honoraria from and served as a consultant for Gilead, Novartis, Glaxo Smith Kline, Janssen, Roche, and received research funding from Infinity, Roche. MM has received honoraria from and served in a consultancy role for Roche, Gilead, and Janssen, and received honoraria from Novartis. SDV and CM have no relevant conflicts of interest to disclose. JMP has served as a consultant for Gilead and Pharmacyclics, and received research funding from Pharmacyclics, AbbVie, and TG Therapeutics. TM has received honoraria from Pharmacyclics, Gilead, and Alexion and has served as a consultant for Morphosys. JAB has served as a consultant for Janssen, Portola, has received research funding from Gilead, Pharmacyclics, and reimbursement from Janssen, Roche. DC is employed by Pharmacyclics and has stock/ownership in Gilead and AbbVie. JL, LG, BC, GC, EH, DFJ are employed by Pharmacyclics and have stock/ownership in AbbVie. JCByrd has no relevant conflict of interest to disclose.

\section{ACKNOWLEDGEMENTS}

Manuscript developed with editorial support from Nexus GG Science LLC funded by Pharmacyclics LLC, an AbbVie Company. The study was sponsored by Pharmacyclics. All authors and their research teams collected the data. Pharmacyclics designed the study, confirmed the accuracy of the data, and compiled it for analysis. The authors had full access to the data and analyses for the compilation of this report. JRB wrote the first draft of this manuscript, which was subsequently reviewed, modified, and approved in its final version by all authors. All authors vouch for the accuracy and completeness of the data reported and the fidelity of the study to the protocol (previously published at NEJM.org).

\section{AUTHOR CONTRIBUTIONS}

JRB wrote the first draft of the manuscript and all authors carefully reviewed the manuscript and approved the final version. The corresponding author had full access to all of the data and final responsibility to submit for publication.

\section{REFERENCES}

1 Catovsky D, Richards S, Matutes E, Oscier D, Dyer MJ, Bezares RF et al. Assessment of fludarabine plus cyclophosphamide for patients with chronic lymphocytic leukaemia (the LRF CLL4 Trial): a randomised controlled trial. Lancet 2007; 370: 230-239.

2 Flinn IW, Neuberg DS, Grever MR, Dewald GW, Bennett JM, Paietta EM et al. Phase III trial of fludarabine plus cyclophosphamide compared with fludarabine for patients with previously untreated chronic lymphocytic leukemia: US Intergroup Trial E2997. J Clin Oncol 2007; 25: 793-798.

3 Hallek M, Fischer K, Fingerle-Rowson G, Fink AM, Busch R, Mayer J et al. Addition of rituximab to fludarabine and cyclophosphamide in patients with chronic lymphocytic leukaemia: a randomised, open-label, phase 3 trial. Lancet 2010; 376: 1164-1174.

4 Keating MJ, O'Brien S, Albitar M, Lerner S, Plunkett W, Giles F et al. Early results of a chemoimmunotherapy regimen of fludarabine, cyclophosphamide, and rituximab as initial therapy for chronic lymphocytic leukemia. J Clin Oncol 2005; 23: 4079-4088.

5 Thompson PA, Tam CS, O'Brien SM, Wierda WG, Stingo F, Plunkett W et al. Fludarabine, cyclophosphamide, and rituximab treatment achieves long-term disease-free survival in IGHV-mutated chronic lymphocytic leukemia. Blood 2016; 127: 303-309.

6 Zenz T, Busch R, Fink A, Winkler D, Fischer K, Bühler A et al. Genetics of patients with F-refractory CLL or early relapse after FC or FCR: Results from the CLL8 trial of the GCLLSG. Blood 2010; 116: 2427.

7 Tam CS, O'Brien S, Plunkett W, Wierda W, Ferrajoli A, Wang X et al. Long-term results of first salvage treatment in CLL patients treated initially with $F C R$ (fludarabine, cyclophosphamide, rituximab). Blood 2014; 124: 3059-3064. 
8 Fried AJ, Bonilla FA. Pathogenesis, diagnosis, and management of primary antibody deficiencies and infections. Clin Microbiol Rev 2009; 22: 396-414.

9 Honigberg LA, Smith AM, Sirisawad M, Verner E, Loury D, Chang B et al. The Bruton tyrosine kinase inhibitor PCl-32765 blocks B-cell activation and is efficacious in models of autoimmune disease and B-cell malignancy. Proc Natl Acad Sci USA 2010; 107: 13075-13080.

10 Byrd JC, Brown JR, O'Brien S, Barrientos JC, Kay NE, Reddy NM et al. Ibrutinib versus ofatumumab in previously treated chronic lymphoid leukemia. $N$ Engl J Med 2014; 371: 213-223.

11 O'Brien S, Jones JA, Coutre SE, Mato AR, Hillmen P, Tam C et al. Ibrutinib for patients with relapsed or refractory chronic lymphocytic leukaemia with $17 p$ deletion (RESONATE-17): a phase 2, open-label, multicentre study. Lancet Oncol 2016; 17: 1409-1418.

12 Trbusek M, Smardova J, Malcikova J, Sebejova L, Dobes P, Svitakova M et al. Missense mutations located in structural p53 DNA-binding motifs are associated with extremely poor survival in chronic lymphocytic leukemia. J Clin Oncol 2011; 29: 2703-2708.

13 Puente XS, Pinyol M, Quesada V, Conde L, Ordóñez GR, Villamor N et al. Wholegenome sequencing identifies recurrent mutations in chronic lymphocytic leukaemia. Nature 2011; 475: 101-105.

14 Quesada V, Conde L, Villamor N, Ordóñez GR, Jares P, Bassaganyas L et al. Exome sequencing identifies recurrent mutations of the splicing factor SF3B1 gene in chronic lymphocytic leukemia. Nat Genet 2011; 44: 47-52.

15 Wang L, Lawrence MS, Wan Y, Stojanov P, Sougnez C, Stevenson K et al. SF3B1 and other novel cancer genes in chronic lymphocytic leukemia. $N$ Engl J Med 2011; 365: 2497-2506

16 Rossi D, Fangazio M, Rasi S, Vaisitti T, Monti S, Cresta S et al. Disruption of BIRC3 associates with fludarabine chemorefractoriness in TP53 wild-type chronic lymphocytic leukemia. Blood 2012; 119: 2854-2862.

17 Foà R, Del Giudice I, Guarini A, Rossi D, Gaidano G. Clinical implications of the molecular genetics of chronic lymphocytic leukemia. Haematologica 2013; 98: 675-685.

18 Stilgenbauer S, Schnaiter A, Paschka P, Zenz T, Rossi M, Döhner K et al. Gene mutations and treatment outcome in chronic lymphocytic leukemia: results from the CLL8 trial. Blood 2014; 123: 3247-3254.

19 Tausch E, Beck P, Schlenk R, Kless S, Galler C, Hillmen P et al. NOTCH1 mutation and treatment outcome in CLL patients treated with chlorambucil (Chl) or ofatumumab-Chl (O-Chl): Results from the phase III study complement 1 (OMB110911). Blood 2013; 122: 257.

20 Rossi D, Rasi S, Fabbri G, Spina V, Fangazio M, Forconi F et al. Mutations of NOTCH1 are an independent predictor of survival in chronic lymphocytic leukemia. Blood 2012; 119: 521-529.

21 Villamor N, Conde L, Martínez-Trillos A, Cazorla M, Navarro A, Beà S et al. NOTCH1 mutations identify a genetic subgroup of chronic lymphocytic leukemia patients with high risk of transformation and poor outcome. Leukemia 2013; 27: 1100-1106.

22 Frampton GM, Fichtenholtz A, Otto GA, Wang K, Downing SR, He J et al. Development and validation of a clinical cancer genomic profiling test based on massively parallel DNA sequencing. Nat Biotechnol 2013; 31: 1023-1031.

23 Rossi D, Bruscaggin A, Spina V, Rasi S, Khiabanian H, Messina M et al. Mutations of the SF3B1 splicing factor in chronic lymphocytic leukemia: association with progression and fludarabine-refractoriness. Blood 2011; 118: 6904-6908.

24 Yu L, Kim HT, Kasar SN, Benien P, Du W, Hoang K et al. Survival of del17p CLL depends on genomic complexity and somatic mutation. Clin Cancer Res 2017; 23: 735-745.

25 Woyach JA, Smucker K, Smith LL, Lozanski A, Zhong Y, Ruppert AS et al. Prolonged lymphocytosis during ibrutinib therapy is associated with distinct molecular characteristics and does not indicate a suboptimal response to therapy. Blood 2014; 123: 1810-1817.

26 Herman SE, Niemann CU, Farooqui M, Jones J, Mustafa RZ, Lipsky A et al. Ibrutinibinduced lymphocytosis in patients with chronic lymphocytic leukemia: correlative analyses from a phase II study. Leukemia 2014; 28: 2188-2196.

27 Byrd JC, Furman RR, Coutre SE, Burger JA, Blum KA, Coleman M et al. Three-year follow-up of treatment-naive and previously treated patients with CLL and SLL receiving single-agent ibrutinib. Blood 2015; 125: 2497-2506.
28 Burger JA, Tedeschi A, Barr PM, Robak T, Owen C, Ghia P et al. Ibrutinib as initial therapy for patients with chronic lymphocytic leukemia. N Engl J Med 2015; 373 2425-2437.

29 Maddocks KJ, Ruppert AS, Lozanski G, Heerema NA, Zhao W, Abruzzo L et al. Etiology of ibrutinib therapy discontinuation and outcomes in patients with chronic lymphocytic leukemia. JAMA Oncol 2015; 1: 80-87.

30 Kazianka L, Drucker C, Skrabs C, Thomas W, Melchardt T, Struve S et al. Ristocetininduced platelet aggregation for monitoring of bleeding tendency in CLL treated with ibrutinib. Leukemia 2016; 31: 1117-1122.

31 Kamel S, Horton L, Ysebaert L, Levade M, Burbury K, Tan S et al. Ibrutinib inhibits collagen-mediated but not ADP-mediated platelet aggregation. Leukemia 2015; 29: 783-787.

32 Thompson PA, Lévy V, Tam CS, Al Nawakil C, Goudot FX, Quinquenel A et al. Atrial fibrillation in CLL patients treated with ibrutinib. An international retrospective study. Br J Haematol 2016; 175: 462-466.

33 Jain P, Keating M, Wierda W, Estrov Z, Ferrajoli A, Jain N et al. Outcomes of patients with chronic lymphocytic leukemia after discontinuing ibrutinib. Blood 2015; 125: 2062-2067.

34 Winqvist M, Asklid A, Andersson PO, Karlsson K, Karlsson C, Lauri B et al. Realworld results of ibrutinib in patients with relapsed or refractory chronic lymphocytic leukemia: data from 95 consecutive patients treated in a compassionate use program. A study from the Swedish Chronic Lymphocytic Leukemia Group. Haematologica 2016; 101: 1573-1580.

35 Chanan-Khan A, Cramer P, Demirkan F, Fraser G, Silva RS, Grosicki S et al. HELIOS investigators. Ibrutinib combined with bendamustine and rituximab compared with placebo, bendamustine, and rituximab for previously treated chronic lymphocytic leukaemia or small lymphocytic lymphoma (HELIOS): a randomised, double-blind, phase 3 study. Lancet Oncol 2016; 17: 200-211.

36 Parikh SA, Kay NE, Shanafelt TD. How we treat Richter syndrome. Blood 2014; 123 : 1647-1657.

37 Fischer K, Bahlo J, Fink AM, Goede V, Herling CD, Cramer P et al. Long-term remissions after FCR chemoimmunotherapy in previously untreated patients with CLL: updated results of the CLL8 trial. Blood 2016; 127: 208-215.

38 Benjamini O, Jain P, Trinh L, Qiao W, Strom SS, Lerner S et al. Second cancers in patients with chronic lymphocytic leukemia who received frontline fludarabine, cyclophosphamide and rituximab therapy: distribution and clinical outcomes. Leuk Lymphoma 2015; 56: 1643-1650.

39 Rossi D, Khiabanian H, Spina V, Ciardullo C, Bruscaggin A, Fama R et al. Clinical impact of small TP53 mutated subclones in chronic lymphocytic leukemia. Blood 2014; 123: 2139-2147.

40 Zenz T, Eichhorst B, Busch R, Denzel T, Häbe S, Winkler D et al. TP53 mutation and survival in chronic lymphocytic leukemia. J Clin Oncol 2010; 28: 4473-4479.

41 Jones J, Coutre S, Byrd JC, Furman R, Hillmen P, Mato A et al. Evaluation of 243 patients with deletion 17p chronic lymphocytic leukemia treated with ibrutinib: a cross-study analysis of treatment outcomes. Presented at: $21^{\text {st }}$ Congress of the European Hematology Association; June 9-12, 2016; Copenhagen, Denmark, Abstract S429.

42 Thompson PA, O'Brien SM, Wierda WG, Ferrajoli A, Stingo F, Smith SC et al. Complex karyotype is a stronger predictor than del(17p) for an inferior outcome in relapsed or refractory chronic lymphocytic leukemia patients treated with ibrutinib-based regimens. Cancer 2015; 121: 3612-3621.

43 Nadeu F, Delgado J, Royo C, Baumann T, Stankovic T, Pinyol M et al. Clinical impact of clonal and subclonal TP53, SF3B1, BIRC3, NOTCH1, and ATM mutations in chronic lymphocytic leukemia. Blood 2016; 127: 2122-2130.

(i) $($ ) NonCommercial-NoDerivs 4.0 International License. The images or other third party material in this article are included in the article's Creative Commons license, unless indicated otherwise in the credit line; if the material is not included under the Creative Commons license, users will need to obtain permission from the license holder to reproduce the material. To view a copy of this license, visit http:// creativecommons.org/licenses/by-nc-nd/4.0/

(c) The Author(s) 2018

Supplementary Information accompanies this paper on the Leukemia website (http://www.nature.com/leu) 